\title{
Breast carcinoma developing in patients on hormone replacement therapy: a histological and immunohistological study
}

\author{
Isobel Fitzgerald O’Connor, Madhuri V Shembekar, Sami Shousha
}

\begin{abstract}
Aim-To study the histopathological features of breast carcinoma developing in postmenopausal patients on hormone replacement therapy (HRT).

Methods-The sample comprised 60 patients with invasive breast carcinoma including 31 who had received HRT at or shortly before presentation, and 29 who had not. Details concerning their tumour size, histological type and grade, lymph node status, and oestrogen and progesterone receptor status were compared. Immunoperoxidase staining for Bcl-2, p53, and E-cadherin was carried out on paraffin sections of all 60 patients. The results were then statistically analysed.

Results-Tumours detected in HRT patients were significantly smaller (mean 17 $\mathrm{mm} v 25 \mathrm{~mm} ; \mathrm{p}=0.0156$ ) and of a lower histological grade $(p=0.0414)$ than those detected in non-HRT patients. The incidence of invasive lobular carcinoma was slightly higher in HRT patients $(19 \% v$ $14 \%$ ). Immunohistologically, $87 \%$ of HRT tumours were Bcl-2 positive (compared with $79 \%$ in the control group), $29 \%$ were p53 positive (45\% in the control), and $48 \%$ were $E$-cadherin positive $(72 \%$ in the control group). Although the differences were not statistically significant there was a trend towards higher incidence of p53 negative and $E$-cadherin negative tumours in HRT patients.

Conclusions-Breast carcinomas detected in patients on HRT have a significantly higher incidence of two favourable prognostic features (small size and a low histological grade). They also show a trend, statistically not significant, of being p53 negative and E-cadherin negative; this may be related to the slightly higher incidence of invasive lobular tumours in these patients.

(F Clin Pathol 1998;51:935-938)
\end{abstract}

Keywords: breast carcinoma; hormone replacement therapy; Bcl-2; p53; E-cadherin

Hormone replacement therapy (HRT) is being increasingly prescribed for the relief of postmenopausal symptoms and to protect patients against coronary heart disease and osteoporosis. The intake of HRT is probably associated with only a slightly increased risk of developing breast carcinoma, ${ }^{1}$ but as the number of women taking HRT increases, there is an increasing number of patients presenting with breast carcinoma who are on HRT. Our aim in this study was to determine whether breast carcinoma developing in patients on HRT has any particular histopathological features. Various histological and immunohistological indices were used to study and compare tumours from two groups of patients, one with and one without a history of HRT.

\section{Methods}

Sixty patients with primary invasive breast carcinoma were included in the study. These patients were selected consecutively from the department of histopathology archives on fulfilling specific criteria. These included age between 48 and 60 years, the availability of patients' notes and tumour paraffin blocks, and whether it was clearly indicated in the patients' notes either that they were definitely not on HRT, or that they were, or definitely had been, on HRT. All patients' notes were obtained from the medical records department at Charing Cross Hospital and individually reviewed.

Information about each patient's histological diagnosis, tumour size and grade, axillary lymph node status, and oestrogen and progestagen receptor status-as determined immunohistochemically ${ }^{2}$ - was obtained from the histopathology reports. Almost all the cases included in this study were originally seen and reported by one of us (SS).

Tumour size was represented by the largest diameter of the tumour on macroscopic examination, unless it was less than $10 \mathrm{~mm}$, when the largest diameter was determined by microscopic examination. Tumour grading was carried out using the grading system of Elston and Ellis. ${ }^{3}$

Each patient's collection of archival haematoxylin and eosin stained sections was then reviewed to confirm the histological diagnosis, and a section representative of the tumour was selected. The corresponding paraffin block was identified and five $5 \mu \mathrm{m}$ thick sections were cut. These were then stained for the tumour markers Bcl-2, p53, and E-cadherin, using the avidin-biotin complex (ABC) immunoperoxidase technique. ${ }^{2}$ Endogenous peroxidase was blocked by a $10 \%$ solution of $30 \%$ hydrogen peroxide. An antigen retrieval step using a pressure cooker was then carried out. ${ }^{2}$ The specific primary antibodies used included mouse Bcl-2 monoclonal antibody (Dako) diluted $1 / 200$ in Tris buffered saline (TBS); mouse monoclonal antibody NCL-p53-D07 (Novocastra Laboratories) diluted $1 / 200$ in TBS; and E-cadherin mouse monoclonal anti- 
Table 1 Histological features of breast carcinomas in patients who had a history of hormone replacement therapy (HRT; 31 cases) and those who did not (29 cases)

\begin{tabular}{llll}
\hline Histological feature & HRT (\%) & Non-HRT (\%) & p Value \\
\hline $\begin{array}{l}\text { Mean size (mm) } \\
\text { Histological type }\end{array}$ & 17 & 25 & 0.0156 \\
$\quad$ Invasive ductal, including mixed & $19(61)$ & $22(76)$ & \\
$\quad$ Invasive lobular & $6(19)$ & $4(14)$ & \\
$\quad \begin{array}{l}\text { Tubular/cribriform } \\
\text { Medullary }\end{array}$ & $5(16)$ & $3(10)$ & \\
Histological grade & $1(3)$ & 0 & \\
$\quad$ grade 1 & $12(39)$ & $4(14)$ & 0.0414 \\
grade 2 & $13(42)$ & $16(55)$ & \\
grade 3 & $6(19)$ & $9(31)$ & \\
\hline
\end{tabular}

body (a gift from Dr Massimo Pignatelli, Hammersmith Hospital, London) used neat. Each antibody was left on the sections for one hour. After washing with TBS, the secondary biotinylated antibody, horse antimouse monoclonal antibody diluted to 1:400 with TBS, was applied for 45 minutes. After another TBS wash, the freshly prepared avidin-biotin complex, diluted $1 / 100$ in TBS, was applied for further 45 minutes. This was then removed by washing with TBS, and freshly prepared diaminobenzedene hydrochloride (DAB) solution was added to the sections for 10 minutes. The slides were then washed in running water, placed in haematoxylin for one minute, washed in running tap water, dipped in a $1 \%$ acid-alcohol solution, and then dehydrated. The slides were then placed in xylene and mounted using coverslips and Starlite mountant (BDH Merk).

Negative and positive controls were used with each staining batch. Positive controls were tumour sections known to be positive for the antigen used. Negative controls were similar sections treated in the same way except for omitting adding the primary antibody.

The staining results were scored semiquantitatively as follows: negative ( -$)$ : no staining, or staining of $<5 \%$ of tumour cells; positive $(+)$ : positive staining of $5-50 \%$ of tumour cells; strongly positive $(++)$ : positive staining of $>50 \%$ of tumour cells.

\section{STATISTICAL ANALYSIS}

Fisher's exact test was used to analyse the data in this study as the sample size was not large and expected values were less than 5 . The null hypothesis is that there is no association between the variable defining the row and the variable defining column. An unpaired $t$ test was also used to identify if there is any statistical significance in the difference in size of the tumours for HRT and non-HRT patients.

Table 2 Axillary lymph node status and immunohistological staining results

\begin{tabular}{llll}
\hline Status & $\begin{array}{l}\text { HRT positive cases/total } \\
\text { examined (\%) }\end{array}$ & $\begin{array}{l}\text { Non-HRT positive cases/total } \\
\text { examined (\%) }\end{array}$ & p Value \\
\hline Node metastasis & $6 / 23(26)$ & $11 / 27(41)$ & 0.1636 \\
OR & $23 / 28(82)$ & $21 / 27(78)$ & $\mathrm{ND}$ \\
PgR & $15 / 28(54)$ & $14 / 27(52)$ & $\mathrm{ND}$ \\
Bcl-2 & $27 / 31(87)$ & $23 / 29(79)$ & 0.5004 \\
p53 & $9 / 31(29)$ & $13 / 29(45)$ & 0.2848 \\
E-cadherin & $15 / 31(48)$ & $21 / 29(72)$ & 0.1319 \\
\hline
\end{tabular}

HRT, hormone replacement therapy; ND, statistical analysis not done as numerical values are similar; OR, oestrogen receptor; PgR, progesterone receptor.

\section{Results}

Thirty one of the consecutive 60 patients were on HRT at the time of presentation or shortly before. A wide variety of HRT preparations was used, and the duration of intake varied between six months and several years. The patients varied in age between 48 and 60 years, with a mean age of 53.7 years. The 29 patients who were not on HRT also had an age range of 48 to 60 years, with a mean of 53.1 years.

Tumour size in the HRT group varied between 4 and $50 \mathrm{~mm}$, with a mean of $17 \mathrm{~mm}$ and a median of $13 \mathrm{~mm}$. The corresponding range, mean, and median values for the control group were $7-55,25$, and $20 \mathrm{~mm}$. The difference between the mean size of each group was statistically significant $(\mathrm{p}=0.0156$, table 1).

Most of the tumours in the two groups were of the invasive ductal type (table 1). The incidence of invasive lobular and the combined group of tubular and cribriform types was higher in the HRT group than in the controls, but the difference was not statistically significant $(\mathrm{p}=0.4986)$.

Twelve patients receiving HRT had a grade 1 invasive carcinoma, and 19 had grade 2 or 3 carcinomas; four patients from the control group had a grade 1 carcinoma and 25 had grade 2 or 3 carcinomas (table 1). Fisher's exact test gives a two sided $\mathrm{p}$ value of 0.0414 , which is considered significant; the relative risk was 1.7368 and the $95 \%$ confidence interval 1.1168 to 2.7011 .

Axillary lymph node dissection was carried out on only 23 of 31 HRT patients and on 27 of 29 non-HRT patients. All patients on whom axillary dissection was not carried out had tumours less than $20 \mathrm{~mm}$ in maximum dimension, and in the majority of these patients the procedure was not considered necessary as the presence of metastases was thought unlikely. For those on whom axillary dissection was carried out, metastases were found in six $(26 \%)$ of 23 HRT patients, and in $11(41 \%)$ of the 27 non-HRT patients (table 2). The difference is not statistically significant $(\mathrm{p}=0.1636)$.

The percentages of oestrogen receptor positive and progesterone receptor positive tumours were similar in the HRT and control groups $(82 \% v 78 \%$ for oestrogen receptor, and $54 \% \vee 52 \%$ for progesterone receptor; table 2).

The percentages of Bcl-2 positively stained tumours were also similar in the two groups ( $87 \%$ and $79 \%$, table $2, \mathrm{p}=0.5004$, NS). On the other hand, although the percentage of p53 positively stained tumours was less in the HRT than the control group $(29 \%$ and $45 \%$, table $2)$, the difference was not significant $(p=0.2848)$. Also, in spite of a lower percentage of E-cadherin positive tumours in the HRT group than in the non-HRT group (48\% v $72 \%$, table 2 ), the $\mathrm{p}$ value was 0.1319 which is not significant.

\section{Discussion}

The findings of this study suggest that exogenous oestrogens in the form of HRT are associated with changes in some histopatho- 
logical features of malignant breast lesions in postmenopausal women.

A history of HRT intake was associated with a significantly higher incidence of smaller tumours. This may simply be a reflection of more rigorous breast screening in these women-for example, Magnusson et al have pointed out that women on HRT have more intense surveillance of their breasts, which would lead to early detection of any tumours that may develop. ${ }^{4}$ However, as most women above the age of 50 in the United Kingdom now undergo regular mammographic breast screening whether they are on HRT or not, the possibility of a direct slowing effect of HRT on tumour growth ought to be considered and investigated.

A higher incidence of non-ductal carcinoma was noted in HRT patients (39\% v 24\% in the control group, table 1), and a statistically significant difference was found in relation to tumour grade (table 1). Fisher's exact test gives a two sided $\mathrm{p}$ value of 0.0414 , which indicates that HRT patients have more lower grade tumours than non-HRT patients. This is in agreement with the findings of Bonnier et al, who also reported that grade 1 tumours and lobular carcinomas were more frequent in patients on HRT than in their counterparts who had not received HRT. ${ }^{5}$ Although this reduced risk of developing high grade tumours may also be the result of greater breast surveillance, it is possible that HRT has a direct action on replication and cellular differentiation in these tumours.

Although there was no statistically significant difference in the prevalence of axillary lymph node metastases in patients who underwent axillary dissection in the two groups $(p=0.1636)$, there was a trend towards a lower incidence of node metastases in HRT patients $(26 \% v 41 \%$, table 2$)$. We should add here that the reason why axillary dissection was not carried out in eight HRT patients, in contrast to only two non-HRT patients, was that they were considered to be at a low risk of developing lymph node metastasis because of the tumour's small size or low malignancy grade. ${ }^{6}$

Our data suggest that HRT has no effect on the expression of oestrogen receptor and progesterone receptor by breast cancer cells (table 2). This contrasts with the findings of Bonnier et al of a higher percentage of hormone receptor negative cases in HRT patients, perhaps because those investigators used a biochemical assay method rather than an immunohistological method as used in our study. Biochemical assays may sometime give false negative results-particularly in small tumours - owing to sampling errors, and immunohistochemical assessment of receptor status is considered more accurate in these lesions. ${ }^{78}$

There was no significant difference between Bcl-2 expression in the two groups of tumours examined $(p=0.5)$. The incidence of $\mathrm{B} \mathrm{cl}-2$ positivity in both groups was relatively high, but was even higher in tumours from the HRT group $(87 \% v 79 \%$, table 2$)$. In this respect, it has recently been reported that $\mathrm{B} \mathrm{cl}-2$ positivity is significantly associated with small tumour size, low tumour grade, non-ductal morphology, oestrogen receptor positivity, and p53 negativity in node negative breast carcinoma. ${ }^{9}$ In our series, most of these features were more common in HRT tumours. In another study, Bcl-2 protein expression was significantly associated with the lobular histological type of breast carcinoma. ${ }^{10}$ In our study all 10 invasive lobular carcinoma (table 1) were Bcl-2 positive. In addition, all eight cases of tubular and cribriform type were also strongly $\mathrm{Bcl}-2$ positive.

Similarly, there was no statistically significant difference between p53 immunostaining of the two tumour groups $(p=0.2848)$, but here there was a more obvious trend towards a much lower incidence of p53 positive tumours in the HRT group ( $29 \%$ v $45 \%$, table 2$)$. This is not surprising in view of the known close association between p53 positivity and higher tumour grade. ${ }^{11} 12$

Most previous studies have found an inverse relation between E-cadherin expression and histological grade of tumour, ${ }^{13}$ and that most invasive lobular carcinomas are E-cadherin negative. ${ }^{1415}$ Of the 334 primary tumours examined by Sitonen et $a l,{ }^{13} 148$ (44\%) were E-cadherin positive, 129 (39\%) had reduced E-cadherin expression, and 56 (15\%) were negative. The corresponding results in all our cases combined were $31 \%$ E-cadherin ++, $31 \%$ E-cadherin+, and $37.9 \%$ negative. The higher negative rate in our study is probably a reflection of the relatively large numbers of invasive lobular carcinomas examined. The lower prevalence of E-cadherin positive tumours in our HRT patients is probably also related to the slightly higher incidence of invasive lobular carcinomas in these patients.

While these results are far from conclusive, it is also not known how many of the HRT patients who have developed breast cancer would have done so if they had not been on HRT. Future investigations could be made into the family history and genetic status of HRT patients with breast carcinoma, to help determine the probability of their developing breast cancer in the absence of HRT. However, as most recent studies indicate that HRT is not associated with a significantly increased risk of developing breast carcinoma, the findings in our investigation tend to suggest that hormone supplementation may provide a beneficial modifying effect on some of the characteristics of tumours developing in patients taking HRT who are destined to develop breast carcinoma. Further investigations are needed to correlate these histopathological results with later outcome and to determine whether molecular mechanisms are involved in these HRT related effects.

We thank Dr Massimo Pignatelli, Hammersmith Hospital, London, for his generous gift of E-cadherin antibody. This work was submitted in part fulfilment of a BSc degree requirements (IFO'C). Dr M V Shembekar's current address is: Department of Histopathology, St Thomas' Hospital, London, UK.

1 Collaborative Group on Hormonal Factors in Breast Cancer. Breast cancer and hormone replacement therapy:
collaborative reanalysis of data from 51 epidemiological 
studies of 52705 women with breast cancer and 108411 women without breast cancer. Lancet 1997;350:1047-59.

2 Shousha S, Peston D. Immunohistochemical demonstration of oestrogen and progesterone receptors in paraffin of oestrogen and progesterone receptors in paraffin sections

3 Elston CW, Ellis IO. Pathological prognostic factors in breast cancer. I. The value of histological grade in breast cancer: experience from a large study with long term follow up. Histopathology 1991;19:403-10.

4 Magnusson C, Holmberg L, Norden T, et al. Prognostic characteristics in breast cancers after hormone replacement therapy. Breast Cancer Res Treat 1996;38:325-34.

5 Bonnier P, Romain S, Giacalone PL, et al. Clinical and biologic prognostic factors in breast cancer diagnosed during postmenopausal hormone replacement therapy. Obstet Gynecol 1995;85:11-17.

6 Barth A, Craig PH, Silverstein MJ. Predictors of axillary lymph node metastases in patients with $\mathrm{T} 1$ breast lymph node metastases in patients

7 Sannino P, Shousha S. Demonstration of oestrogen receptors in paraffin wax sections of breast carcinoma using the monoclonal antibody 1D5 and microwave oven processing. F Clin Pathol 1994;47:90-2.

8 Taylor CR. Paraffin section immunocytochemistry for estrogen receptor. The time has come. Cancer 1996;77: 2419-22
9 Barbareschi M, Caffo O, Vernesi S, et al. Bcl-2 and p53 expression in node-negative breast carcinoma: A study with long-term follow-up. Hum Pathol 1996;27:1149-55.

10 Joensuu H, Pylkkanen L, Toikkanen S. Bcl-2 protein expression and long-term survival in breast cancer. $A m \mathcal{F}$ Pathol 1994;145:1191-8.

11 Domagala W, Harezga B, Szadowska A, et al. Nuclear p53 protein accumulates preferentially in medullary and high-grade ductal but rarely in lobular breast carcinomas. Am f Pathol 1993;142:669-74.

12 Pratap R, Shousha S. Breast carcinoma in women under the age of 50: Relationship between p53 immunostaining, tumor grade and axillary lymph node status. Breast Cancer Res Treat 1998;49:35-9.

13 Sitonen SM, Kononen JT, Helin HJ, et al. Reduced E-cadherin expression is associated with invasiveness and favourable prognosis in breast cancer. Am $\mathcal{F}$ Clin Pathol 1996; 105:394-402.

14 Gamallo C, Palacios J, Suarez A, et al. Correlation of E-cadherin expression with differentiation grade and histological type in breast carcinoma. Am 7 Pathol 1993;142: 987-93.

15 Moll R, Mitze M, Frixen UH, et al. Differential loss of E-cadherin expression in infiltrating ductal and lobular breast carcinomas. Am f Pathol 1993;143:1731-42. 